\title{
Released all-porous-silicon microstructure for spectrometer applications
}

\author{
$\underline{\text { Xiao Sun }}^{1 *}$, Yaman Afandi ${ }^{1,2}$, Giacinta Parish $^{2}$ and Adrian Keating ${ }^{1}$ \\ ${ }^{1}$ Department of Mechanical and Chemical Engineering, University of Western Australia, 35 Stirling Hwy, Crawley, Western \\ Australia 6009, Australia \\ ${ }^{2}$ Department of Electrical, Electronic and Computer Engineering, University of Western Australia, 35 Stirling Hwy, Crawley, \\ Western Australia 6009, Australia \\ *xiao.sun@uwa.edu.au
}

All-porous-silicon microstructures have been released with in-situ controllable current density to achieve low residual stress and out-of-plane stress gradient in the porous silicon (PS) films. Optical transmission of large area released PS structures was investigated through both Fourier-transform infrared spectroscopy (FTIR) and modelling in the near infrared and mid infrared wavelength range.

\section{Introduction}

Microelectromechanical systems (MEMS)-based tuneable Fabry-Perot filters have been developed within various infrared thermal imaging bands [1-3]. For such filters, released distributed Bragg reflectors (DBR) are needed to achieve narrowband transmission with high signal-to-noise ratio and a controllable wavelength. Usually they are fabricated through releasing multilayer materials which form alternate quarter wavelength thick layers of high refractive index and low refractive index. However, this method requires multistep deposition/etching with limited choice of suitable materials in terms of desired refractive index and micromachining compatibility.

Alternatively, through controlling the anodisation current density and HF electrolyte concentration in the formation process of porous silicon, modifying film properties such as porosity, refractive index and optical thickness can be easily achieved. Multilayer PS based distributed Bragg reflectors [4, 5] and optical filter based on released PS [6] have been fabricated, however, the MEMS device performance was limited by the nonuniform porosity and tendency to oxidise (ageing of the PS film), especially in released thick porous silicon devices. Through $\mathrm{N}_{2}$ annealing, oxidation in ambient air of PS films is eliminated and the PS films become robust enough to apply standard photolithography [7], therefore allowing complex and scalable PS-MEMS structures to be fabricated. Methods to tune the residual stress and stress gradient by varying the current density during anodisation have been investigated to achieve a flat surface profile of the released PS microstructures having reduced out-ofplane stress gradient [8].

In this work, suspended PS plate microstructures with a large area of up to $0.36 \mathrm{~mm}^{2}$ have been released, which show promise as a platform for an optical spectrometer. Fourier transform infrared spectroscopy (FTIR) was utilised to measure the transmission of the released PS structure. A transmission spectra model based on released single layer and multilayer PS was built for comparison and analysis. The study shows the opportunity to build large size, transmission controllable PS based optical spectrometers.

\section{Results and discussion}

PS films were fabricated with room temperature anodisation in a $15 \% \mathrm{HF} / \mathrm{ethanol}$ solution, on moderately doped p-type (100) silicon wafers with resistivity of 0.08 $0.12 \Omega \cdot \mathrm{cm}$. The current density during anodisation was decreased from $20 \mathrm{~mA} / \mathrm{cm}^{2}$ to $8 \mathrm{~mA} / \mathrm{cm}^{2}$ over the film forming process, which was designed to compensate the
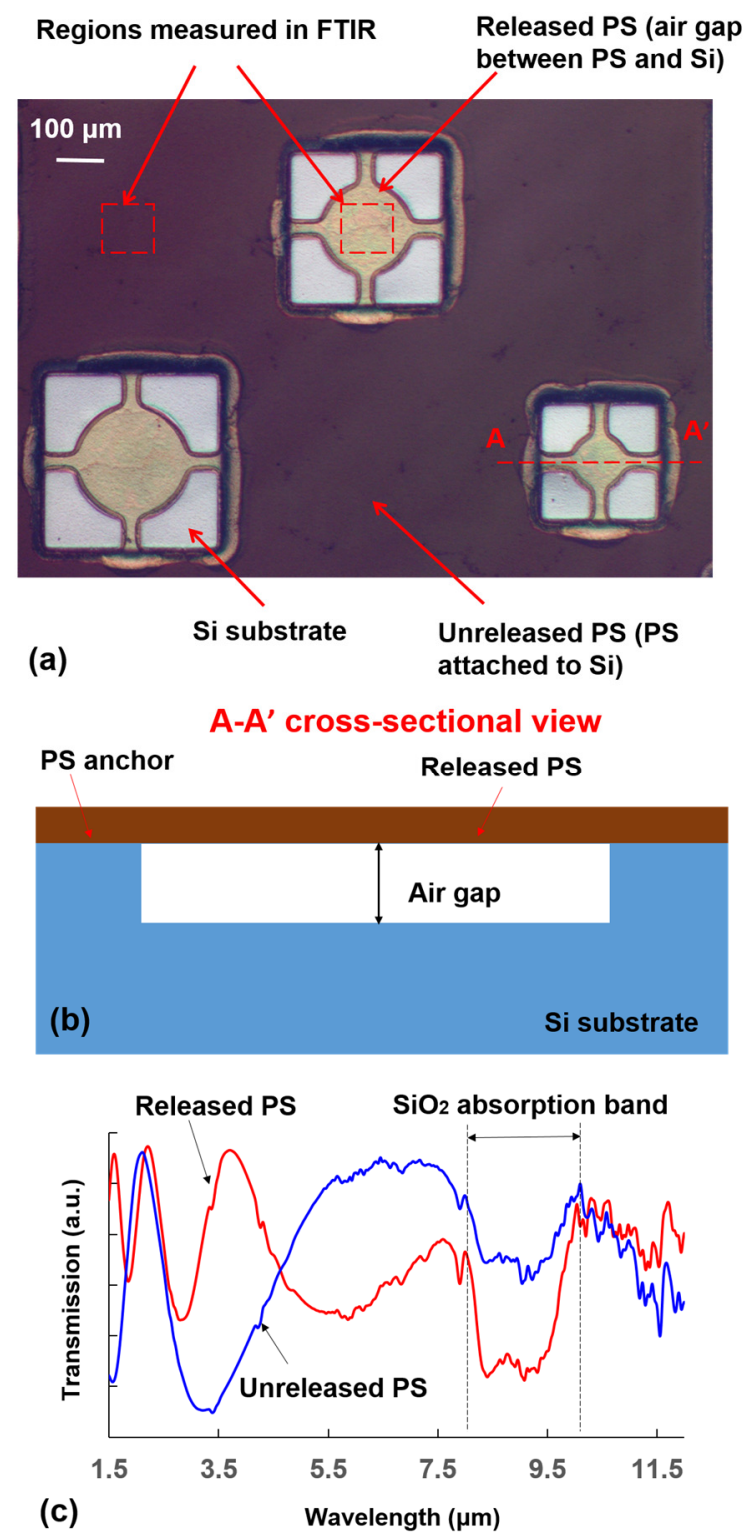

Figure 1: Transmission of released and unreleased PS microstructures. (a) Optical micrograph of released suspended PS plate microstructures; (b) Cross-sectional schematic of A-A' in (a); (c) transmission spectra measured by FTIR, with air gap of $2.9 \mu \mathrm{m}$. 
out-of-plane porosity gradient induced stress gradient [8] and achieve a flat PS microstructure surface. The resulting film has a thickness of $t=2.5 \mu \mathrm{m}$ and porosity $P=77 \%$. After anodisation, the PS was annealed at $600{ }^{\circ} \mathrm{C}$ in nitrogen for $48 \mathrm{~min}$, resulting in a robust film with high resistance to alkaline developer over the entire surface [9]. After annealing, established processes consisted of repeated patterning, RIE and electropolishing, followed by critical point drying, were implemented to release suspended PS plate structures with diameters range from $100 \mu \mathrm{m}$ to $300 \mu \mathrm{m}$ (circular) [10] or side length of 300$600 \mu \mathrm{m}$ (square). The air gap height between the released $\mathrm{PS}$ and $\mathrm{Si}$ substrate can be controlled through electropolishing duration, and was designed to range from $2.5 \mu \mathrm{m}$ to $8 \mu \mathrm{m}$ in this work.

FTIR measurement was carried out with a PerkinElmer Spotlight i200 microscopy system, which can accurately locate the measurement region within an area of $100 \mu \mathrm{m}$ diameter. As shown in Fig.1 (a), suspended PS plate microstructures of different sizes were released with an airgap of $2.9 \pm 0.4 \mu \mathrm{m}$ between the released PS and Si. The cross-sectional view of the released PS microstructures is shown in Fig. 1(b). FTIR transmission was measured from the near infrared to mid infrared range $(1.5-12 \mu \mathrm{m})$ in both released PS regions and unreleased PS regions; the spectra are shown in Fig. 1(c). A clear change of transmission peak positions between the spectra of released PS and unreleased PS was observed, indicating optical resonance resulting from the air gap cavity plays an important role in tuning the transmission peaks.

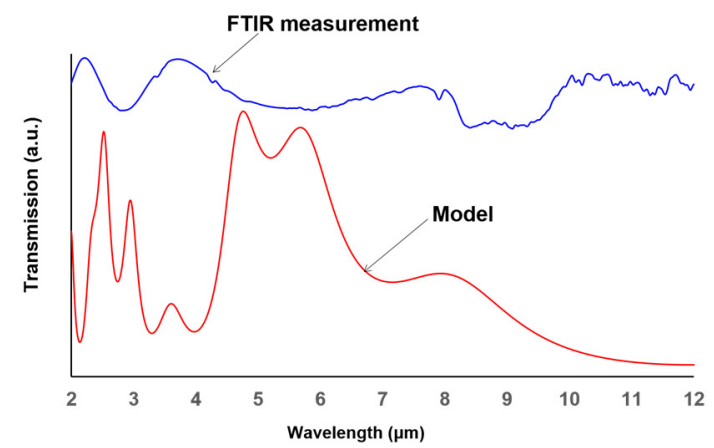

Figure 2: FTIR measurement and model of released single layer PS microstructure transmission spectra. Air gap of $2.9 \mu \mathrm{m}$.

To further understand the transmission of released PS structures, the FTIR measured data was compared with modelling results as shown in Fig. 2. The model was built based on a PS/air/Si/air structure, with PS film of porosity $77 \%$ and an airgap of $2.9 \mu \mathrm{m}$. The measured data showed similar transmission peak variations to the model, however

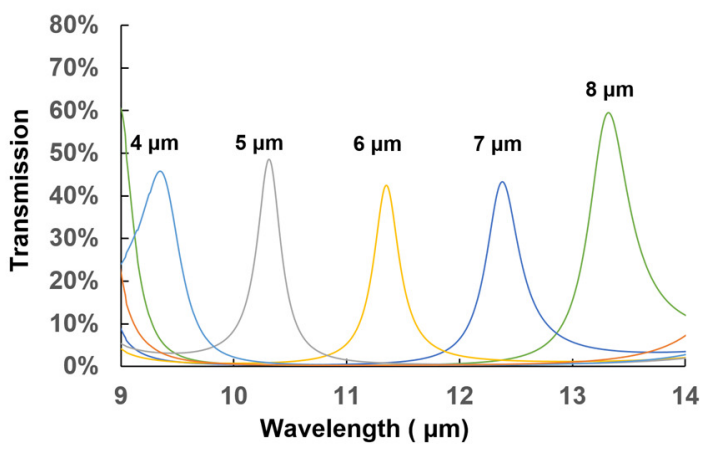

Figure 3: Modelled transmission of multilayer PS DBR filters with airgap ranges from $4 \mu \mathrm{m}$ to $8 \mu \mathrm{m}$. with peak positions at different wavelengths. This could be due to non-flat structures; further topological studies are required to understand the flatness of these structures.

Investigation is ongoing through refinements of setup and background spectrum in FTIR to improve the measurement and signal/noise ratio. However, the work shows a pathway to release large size suspended PS plate microstructures, which provides the opportunity for fabricating multiplayer PS structures to work as DBR filters for an optical spectrometer.

The performance of a modelled narrowband filter based on a 3-layer PS DBR is shown in Fig.3. This model does not include the $\mathrm{SiO}_{2}$ absorption which exists and needs to be eliminated in future structures. With the ability to fabricate multiplayer PS [11] and release large size PS microstructures with different air gaps, complex PS based Fabry-Perot filters should be achievable.

\section{Conclusion}

Large size (area up to $0.36 \mathrm{~mm}^{2}$ ) suspended PS plate microstructures were released, and FTIR transmission was measured and compared with a model. The work provides a pathway for released complex PS filter fabrication, indicating the possibility of PS infrared thermal imaging applications.

\section{Acknowledgements}

This work has been supported by the Australian Research Council under DP170104266. We acknowledge the support from the Western Australian Node of the Australian National Fabrication Facility, and the Office of Science of the Western Australia State Government.

\section{References}

[1] D.K. Tripathi, et al., Silicon-air-silicon distributed Bragg reflectors for visible and near infrared optical MEMS, J. Microelectromech. S., 24(2015) 1245-7.

[2] D.K. Tripathi, et al., Large-area mems-based distributed bragg reflectors for short-wave and mid-wave infrared hyperspectral imaging applications, J. Microelectromech. S., 24(2015) 2136-44

[3] H. Mao, et al., Ge/ZnS-Based Micromachined Fabry-Perot Filters for Optical MEMS in the Longwave Infrared, J. Microelectromech. S., 24(2015) 2109-16.

[4] L. Pavesi, P. Dubos, Random porous silicon multilayers: application to distributed Bragg reflectors and interferential Fabry-Perot filters, Semicond. Sci. and Tech., 12(1997) 570.

[5] H.-J. Kim, et al., A distributed Bragg reflector porous silicon layer for optical interferometric sensing of organic vapor, Sensors and Actuators B: Chemical, 155(2011) 673-8.

[6] G. Lammel, et al., Microspectrometer based on a tunable optical filter of porous silicon, Sensors and Actuators A: Physical, 92(2001) 52-9.

[7] M. Lai, et al., Development of an alkaline-compatible poroussilicon photolithographic process, J. Microelectromech. S., 20(2011) 418-23.

[8] Y. Afandi, et al., Using Current Density to Control Stress and Porosity in Porous Silicon Fabrication, International Conference on Nanoscience and Nanotechnology (ICONN), Wollongong, Australia, 2018.

[9] M. Lai, Porous silicon and its application for micromachining technologies: University of Western Australia; 2011.

[10] X. Sun, Micromachining Based On Porous Silicon: University of Western Australia; 2016.

[11] M. Lai, et al., Multilayer porous silicon diffraction gratings operating in the infrared, Nanoscale research letters, 7(2012) 645. 